\title{
Bariatric Surgery and Bone Loss: Novel Mechanisms and Comparison of Different Modalities
}

Brzozowska MM ${ }^{1}$, Bliuc D ${ }^{1}$, Hong $A^{2}$, Jorgensen ${ }^{3}$, Talbot $M^{3}$, Rigas ${ }^{3}$, Chen $W^{1}$, Pocock NA ${ }^{4}$, Eisman $\mathrm{JA}^{1^{*}}$, White $\mathrm{CP}^{2}$, Baldock PA ${ }^{1,5}$, Center JR ${ }^{1 *}$.

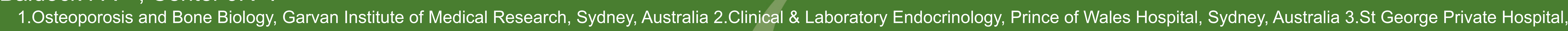
Sydney, Australia 4.Department of Nuclear Medicine, St Vincent's Hospital, Sydney, Australia 5. Neurological Disease Division, Garvan Institute of Medical Research, St Vincent's Hospital, Sydney, Australia.

Introduction

Obesity rates have increased in recent decades

Bariatric surgery remains the most effective therapy for weight loss.

The skeletal consequences of obesity surgery are not clear.

\section{Aims}

To determine:

> Bone loss in obese subjects following weight Bone loss in
loss surgery.

Coss suss in obese subjects following weight

Collowing weight loss.

$>$ impact on BMD

\section{Methods}

Nonrandomized, prospective study of obese patients undergoing weight loss through 3 interventions

$>$ Diet and exercise program (Diet)- followed for 1 year

> Gastric Banding (GB)-followed for 1 year

- Gastric Sleeve (GS)- followed over 2 years

Measurements at all visits:

Anthropometry, biochemistry, gut hormones, adipokines $>\mathrm{Ca}^{++}$and vit $\mathrm{D}$ supplements
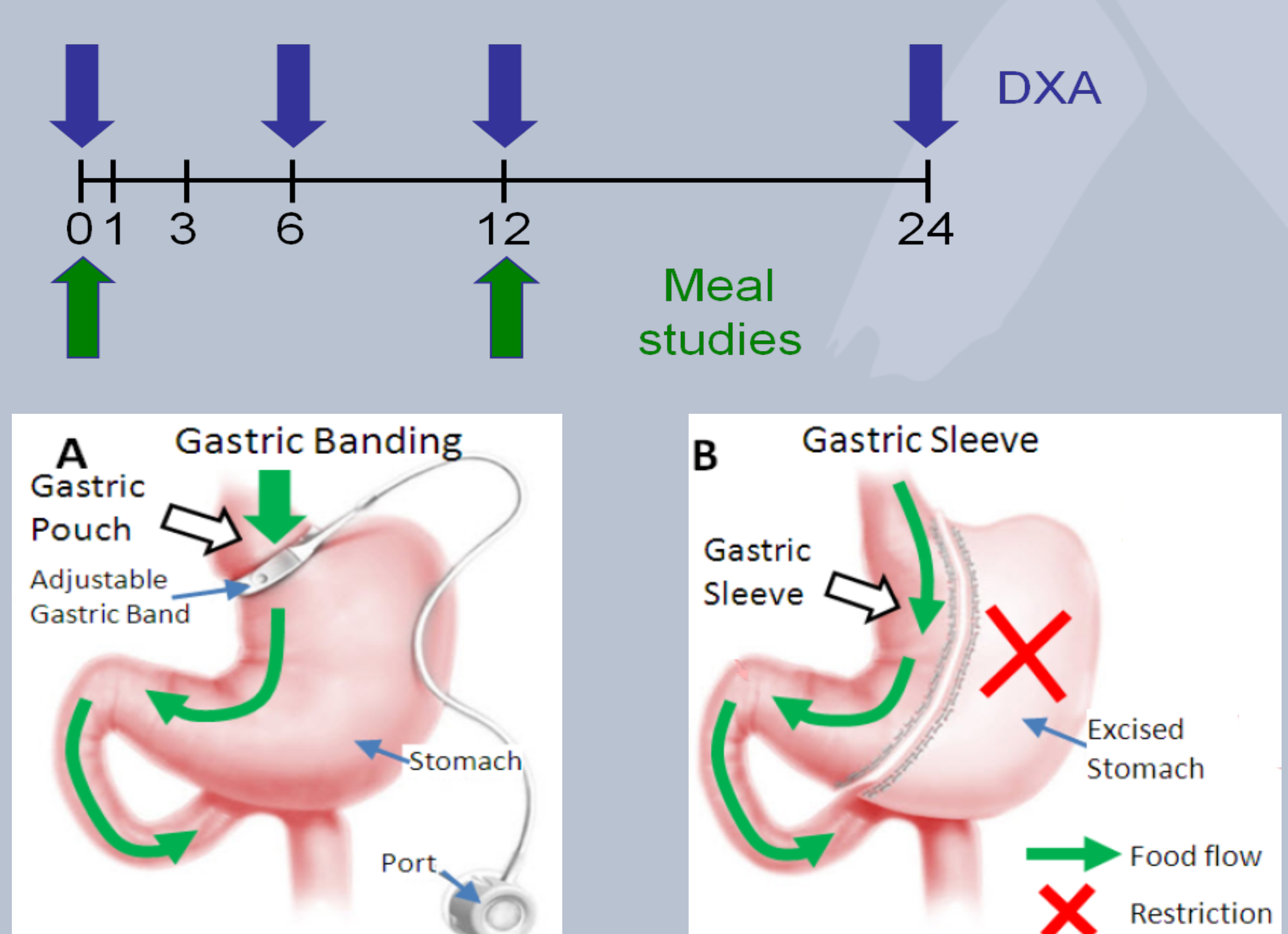

Baseline Characteristics

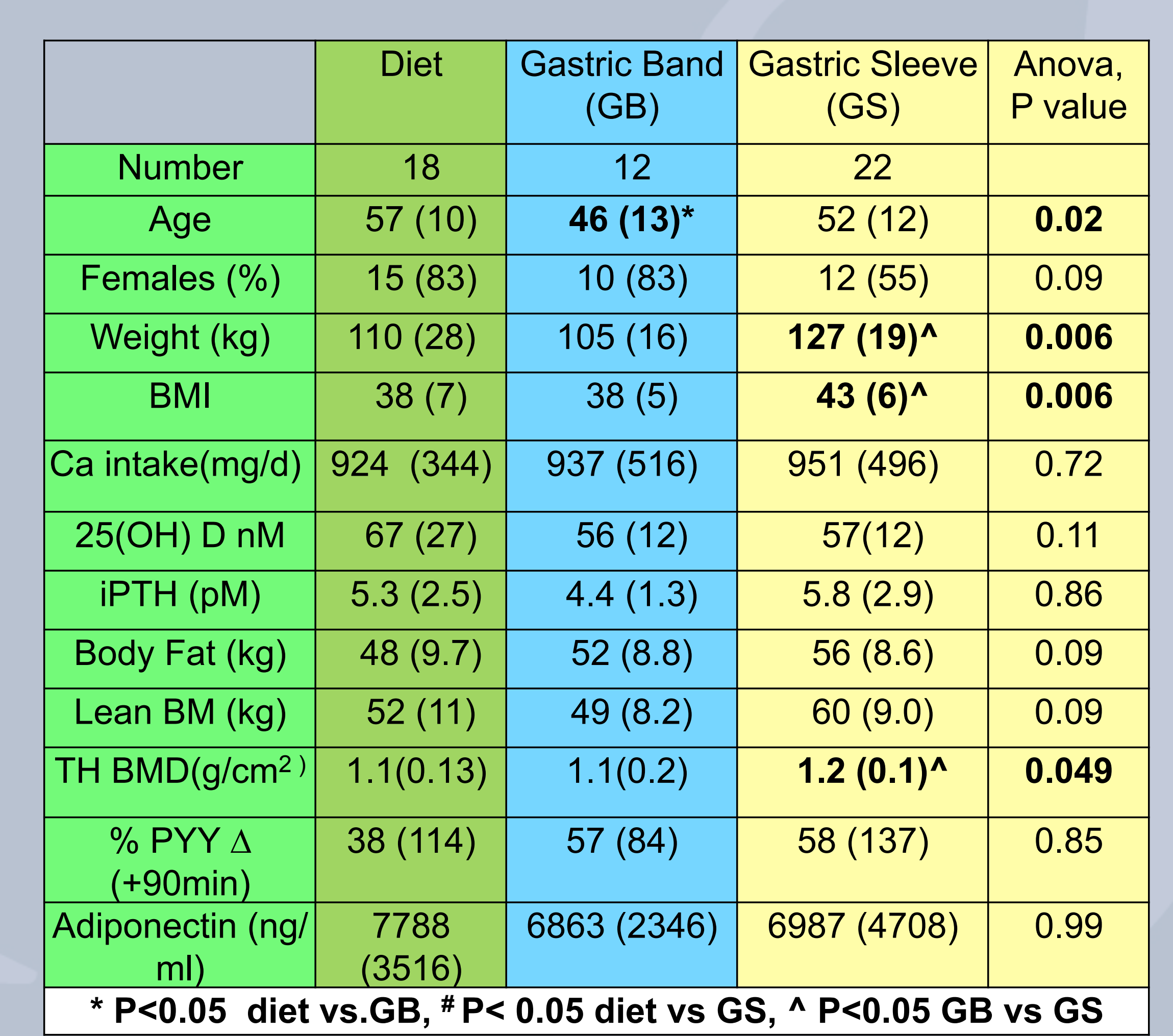

Comparison of study procedures at $12 \mathrm{~m}$

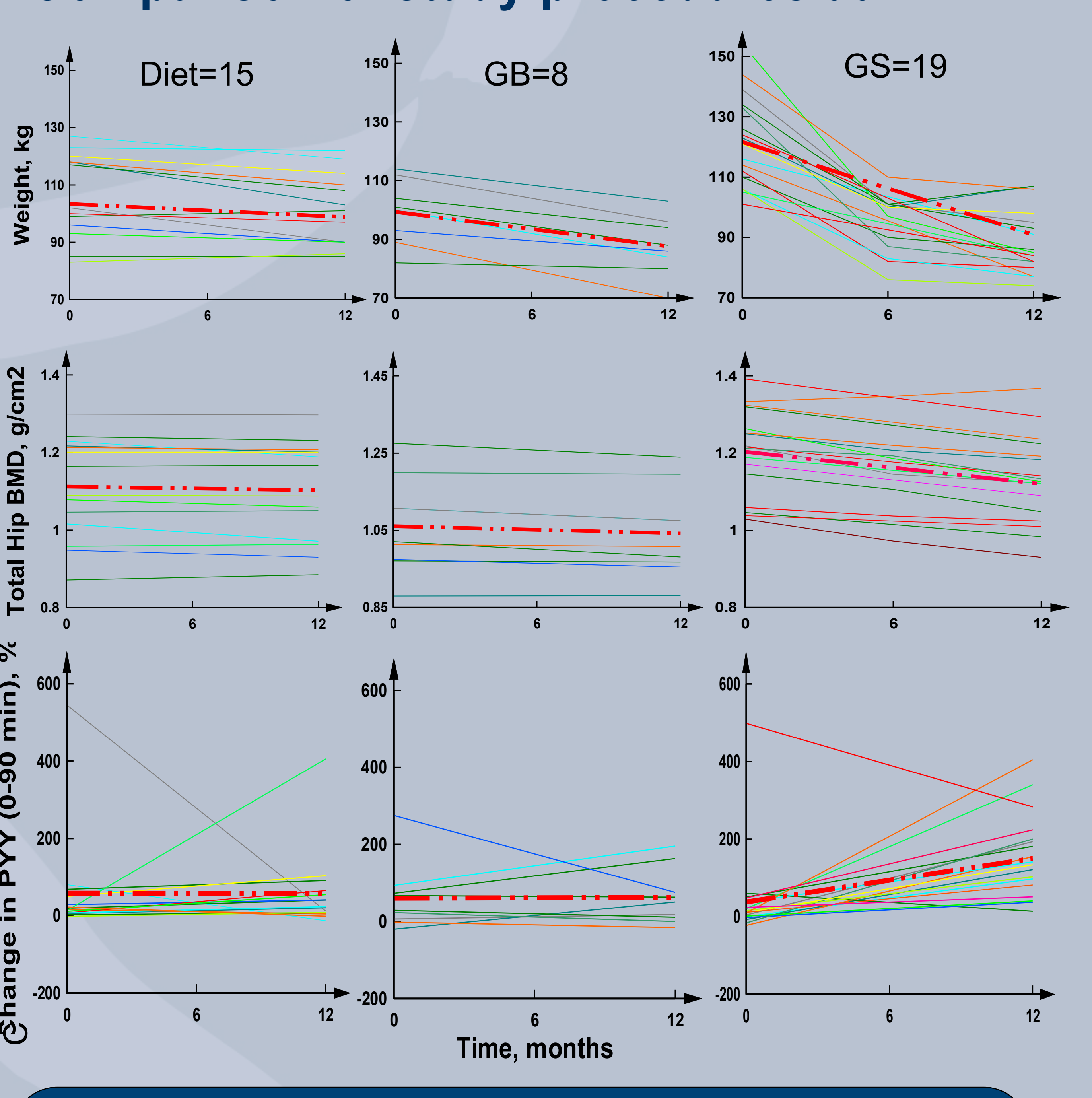

$>$ GS was the most efficient weight loss modality.

$>$ Diet produces modest weight loss.

Bone loss was significant only in GS.

> The postprandial PYY response 12 months post- GS was
significantly higher compared with Diet or GB group.
Comparison of study procedures at 12 months

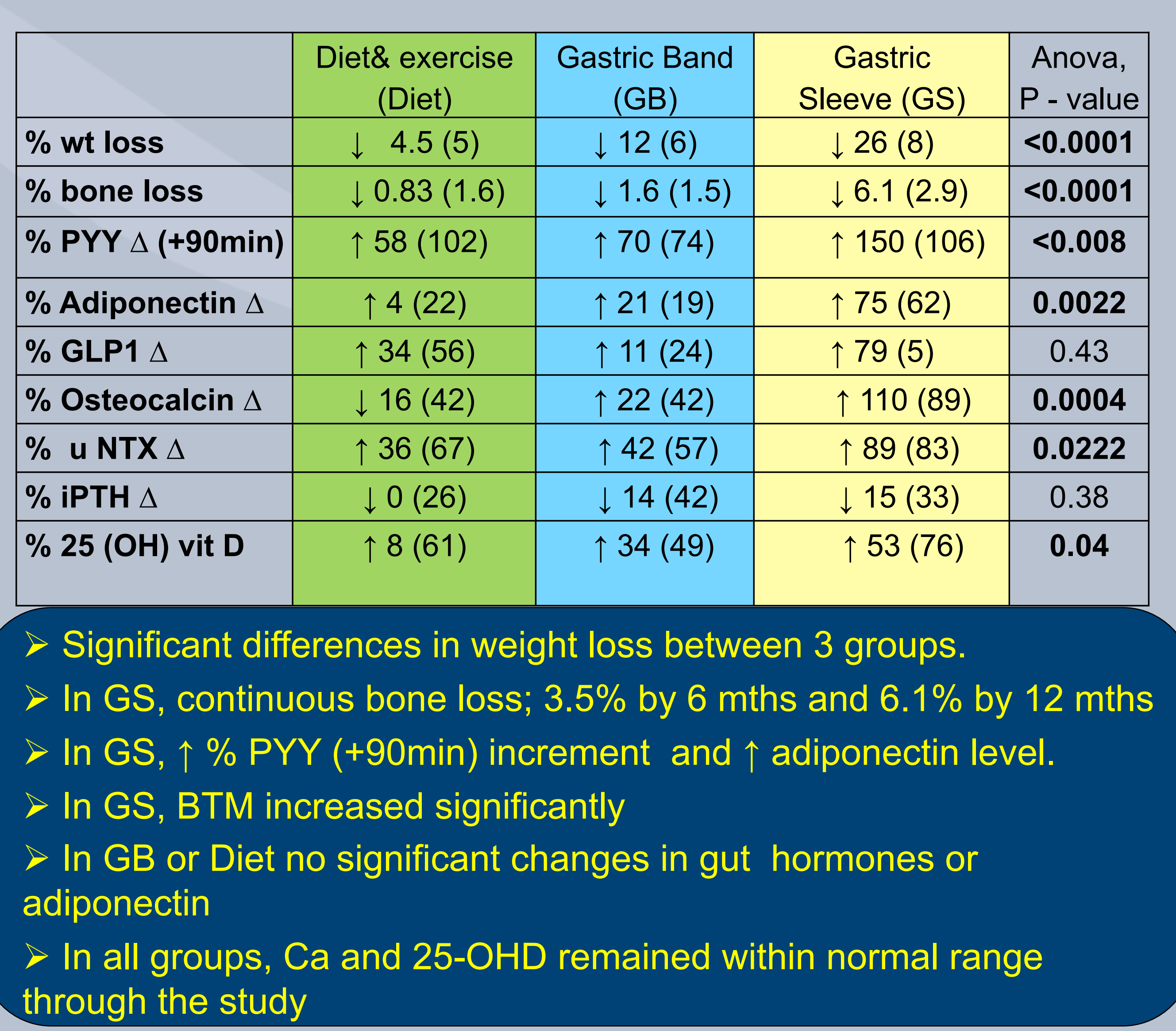

Correlations between weight loss, bone loss, adiponectin and PYY for all patients

Weight loss,
TH BMD
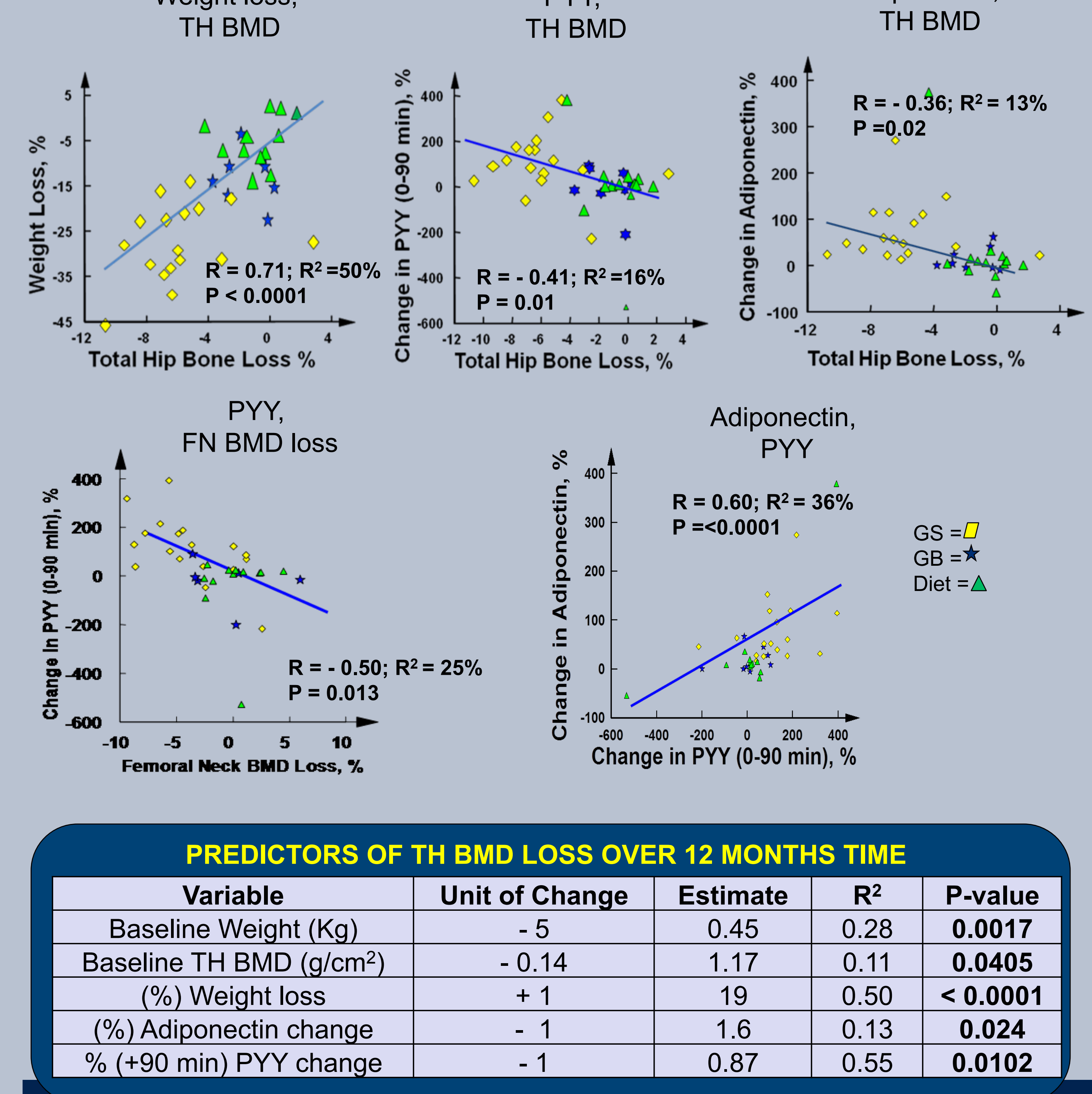

GS: Continuous bone loss over 24months after early weight loss
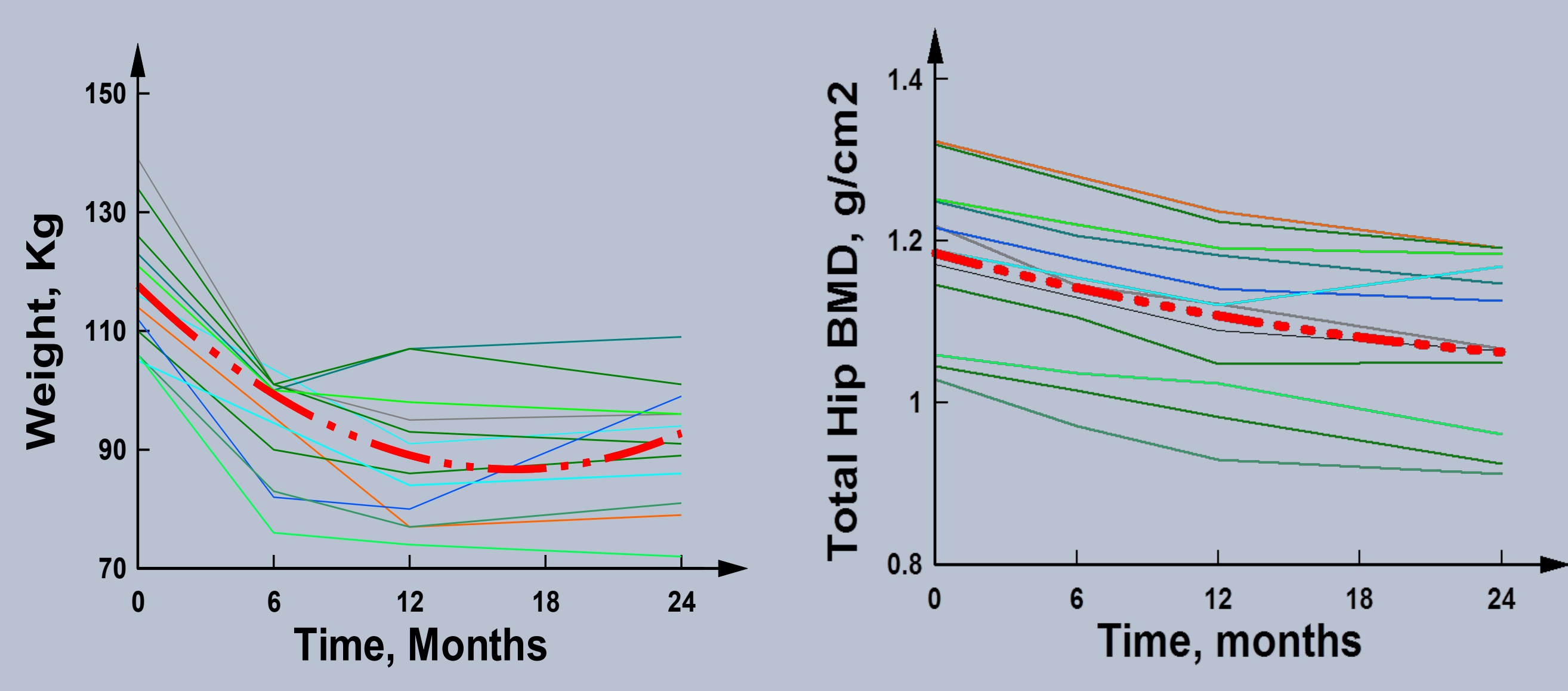

\section{For 12 GS patients with 24 months date}

> Maximum weight loss occurred during the first 6 months

$>$ Bone loss was continuous across the 24 month period

$>$ These patients experienced a further BMD loss, totalling $9(3) \%$

$>$ There was a $\downarrow$ in mean TH T-score from +1.0 to $0.2, p=0.04$

There was a continuous lean mass loss of $13.5 \%$

\section{Conclusion}

$\rightarrow$ GS is the most effective weight loss modality.

Bone loss was the highest in GS patients.

> Bone loss at 12 months was significantly correlated with weight loss,
change in PYY and adiponectin.

$>$ In the multivariate analysis weight loss and postprandial PYY

$>$ The bone loss and lean mass loss in GS group were continuous (24

months), while weight loss only occurred during first 6 months.

> Calcium and 25-OHD were normal throughout this study

These findings have significant clinical implications for people undergoing bariatric surgery

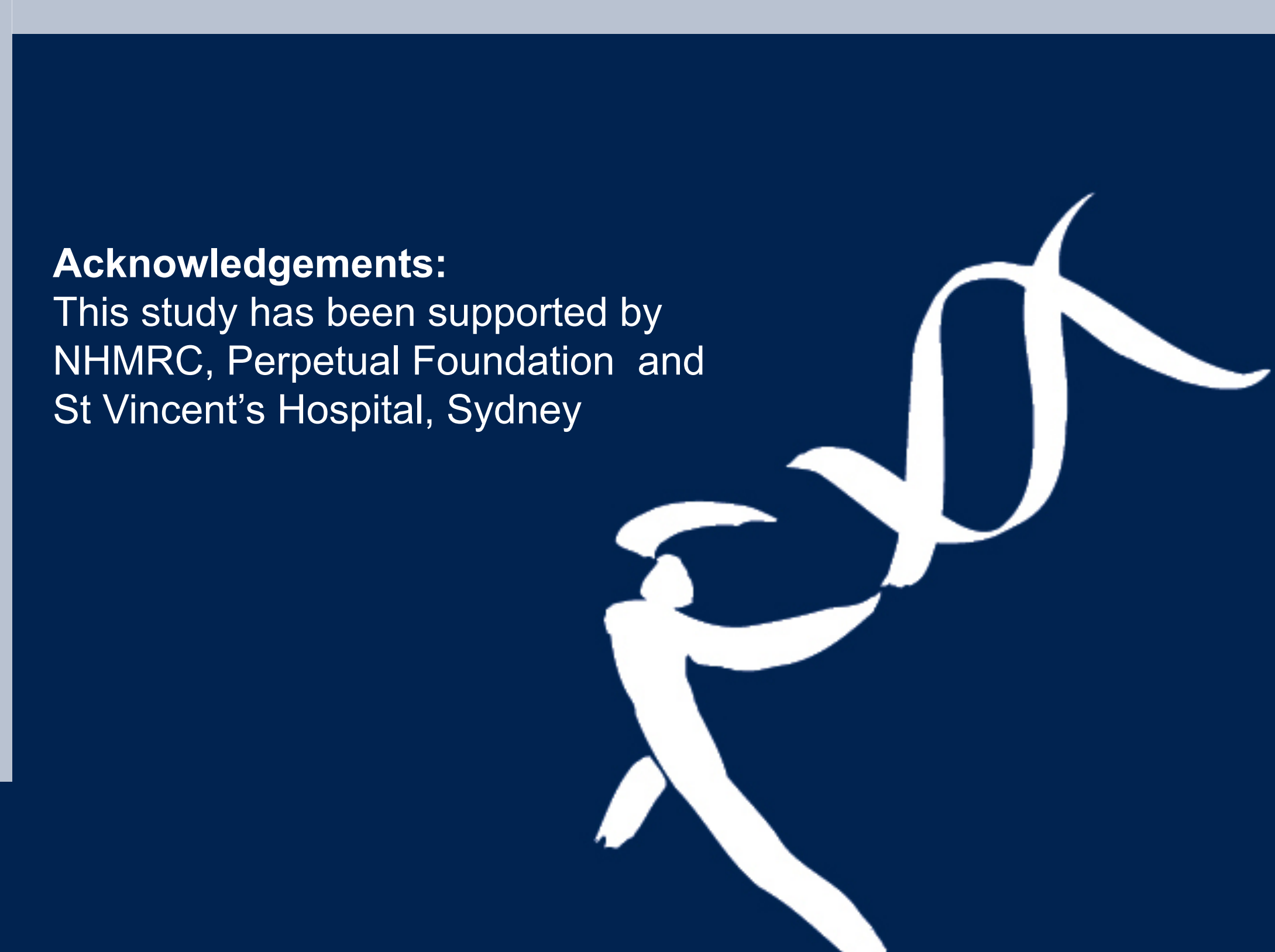

